Supporting Information

\title{
Potential use of serum proteomics for monitoring COVID-19 progression to complement RT-PCR detection
}

Ying Zhang1\#, Xue Cai2,3,4\#, Weigang Ge2,3,4,5\#, Donglian Wang1\#, Guangjun Zhu1\#, Liujia Qian2,3,4\#, Nan Xiang2,3,4,5, Liang Yue2,3,4, Shuang Liang2,3,4, Fangfei Zhang2,3,4, Jing Wang1, Kai Zhou1, Yufen Zheng1, Minjie Lin1, Tong Sun1, Ruyue Lu1, Chao Zhang1, Luang Xu2,3,4, Yaoting Sun2,3,4, Xiaoxu Zhou2,4, Jing Yu2,3,4, Mengge Lyu2,3,4, Bo Shen1, Hongguo Zhu1*, Jiaqin Xu1*, Yi Zhu2,3,4*, Tiannan Guo2,3,4*

1Taizhou Hospital of Zhejiang Province Affiliated to Wenzhou Medical University, Linhai 317000, Zhejiang, China;

2 Key Laboratory of Structural Biology of Zhejiang Province, School of Life Sciences, Westlake University, Xihu District 310000, Hangzhou, Zhejiang, China; 3 Center for Infectious Disease Research, Westlake Laboratory of Life Sciences and Biomedicine, Xihu District 310000, Hangzhou, Zhejiang, China;

4 Institute of Basic Medical Sciences, Westlake Institute for Advanced Study, Xihu District 310000, Hangzhou, Zhejiang, China;

5Westlake Omics (Hangzhou) Biotechnology Co., Ltd. No.1, Yunmeng Road, Cloud Town, Xihu District 310000, Hangzhou, Zhejiang Province, China;

\# co-first;

*Correspondence: zhuhg@enzemed.com; xujq@enzemed.com; zhuyi@westlake.edu.cn; guotiannan@westlake.edu.cn 


\section{Table of Contents}

Figure S1. Quality control of proteomics data acquired with SWATH-MS.

Figure S2. Biological replicates and technical replicates of the proteomics data acquired with SWATH-MS.

Figure S3. Enriched pathways based on 48 significantly dysregulated proteins.

Figure S4. Pearson correlation between protein expression and clinical indexes in the severe and non-severe cases.

Figure S5. Machine learning using data from 144 patients.

Figure S6. Machine learning using data from 53 patients with proteomic data.

Table S1. Details of the SWATH-MS method.

Table S2. Clinical characteristics of 676 samples derived from 144 COVID-19

patients, 24 non-COVID-19 patients, and 21 healthy individuals.

Table S3. Proteomics data of 320 serum samples. 
Figure S1. Quality control of proteomics data acquired by SWATH-MS. Pairwise comparison of eight QC samples was performed with Pearson correlation coefficients shown.

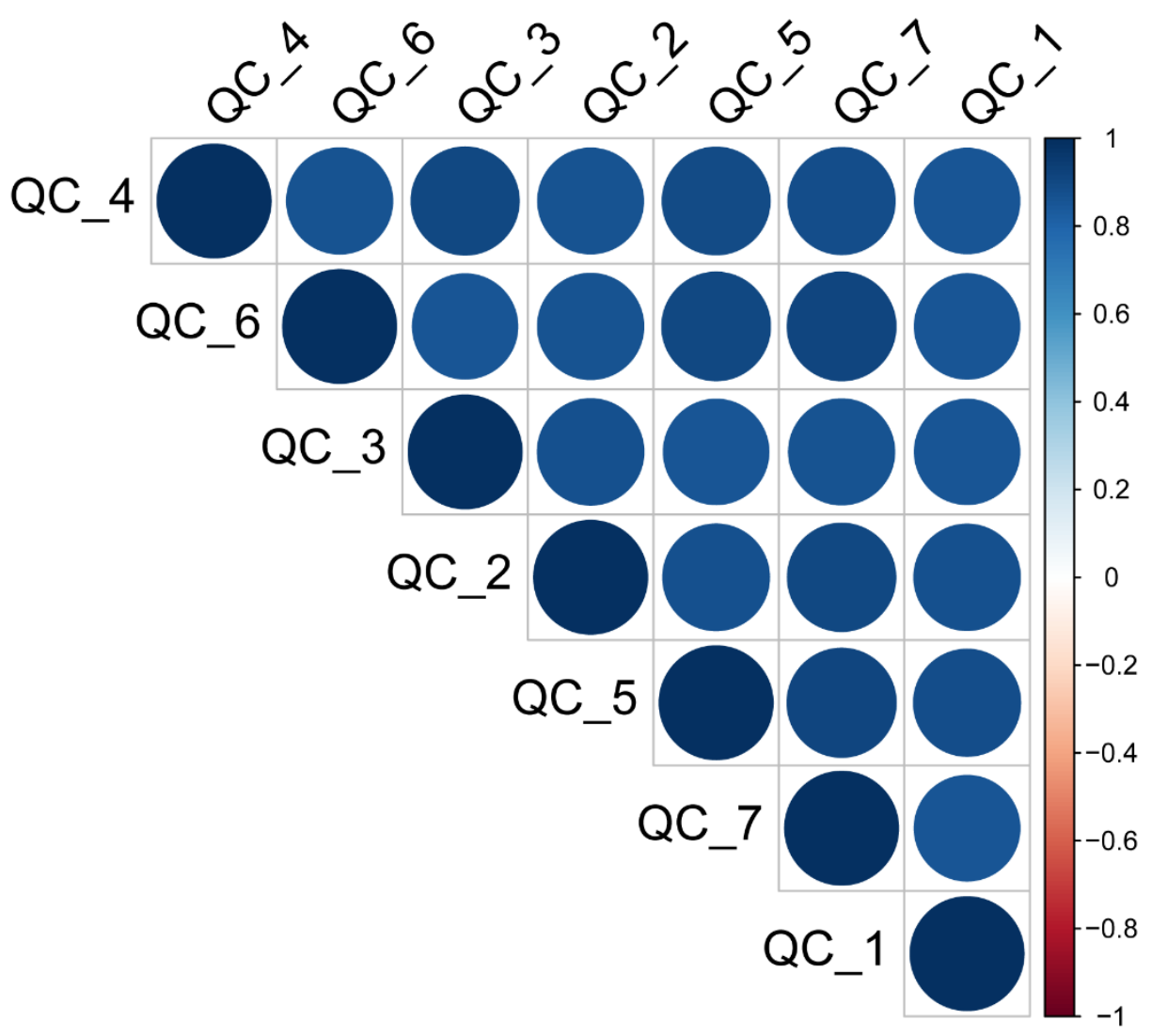


Figure S2. Biological replicates and technical replicates of the proteomics data acquired the SWATH-MS. Violin plots summarizing the Pearson correlation coefficients for pair-wise comparison of 27 biological replicates (left plot) and 22 technical replicates (right plot).

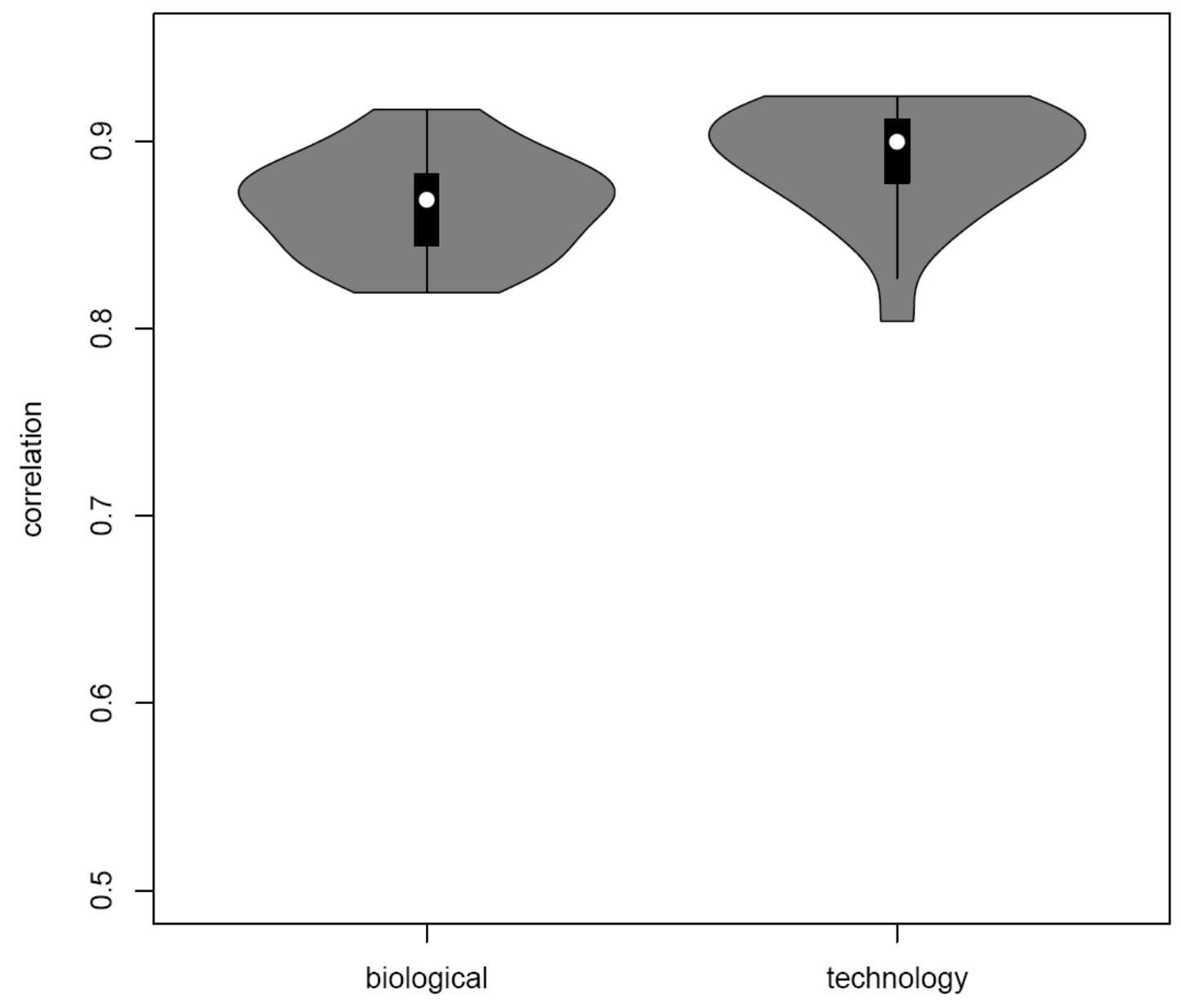


Figure S3. Enriched pathways based on $\mathbf{4 8}$ significantly dysregulated proteins. 25 pathways were enriched in IPA analysis using 48 dysregulated proteins, and nine of them were significantly enriched ( $p$ value $<0.05$ ). The redness of the node is positively correlated to the significance of enrichment.

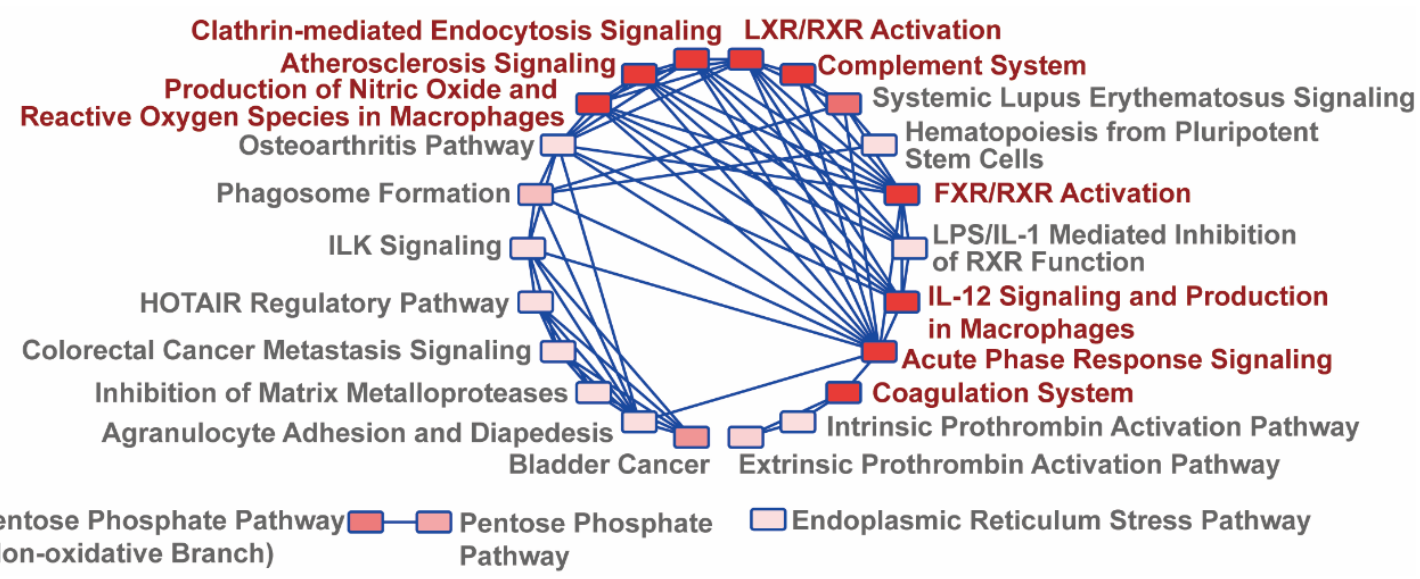


Figure S4. Pearson correlation between protein expression and clinical indexes in the severe and non-severe cases. The $\mathrm{x}$-coordinate represents $\log 2$-transformed protein expression, while the y-coordinate denotes clinical index data.

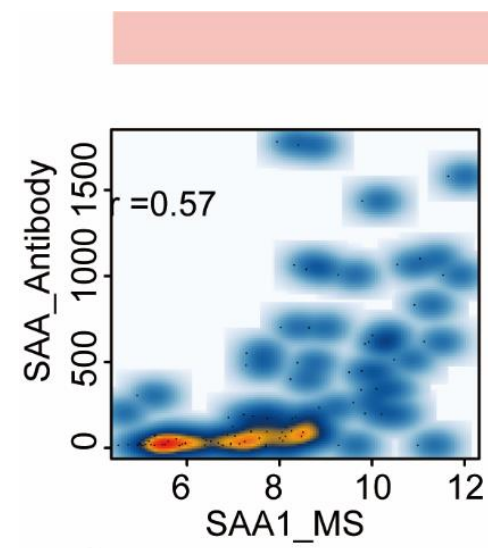

\section{Severe}
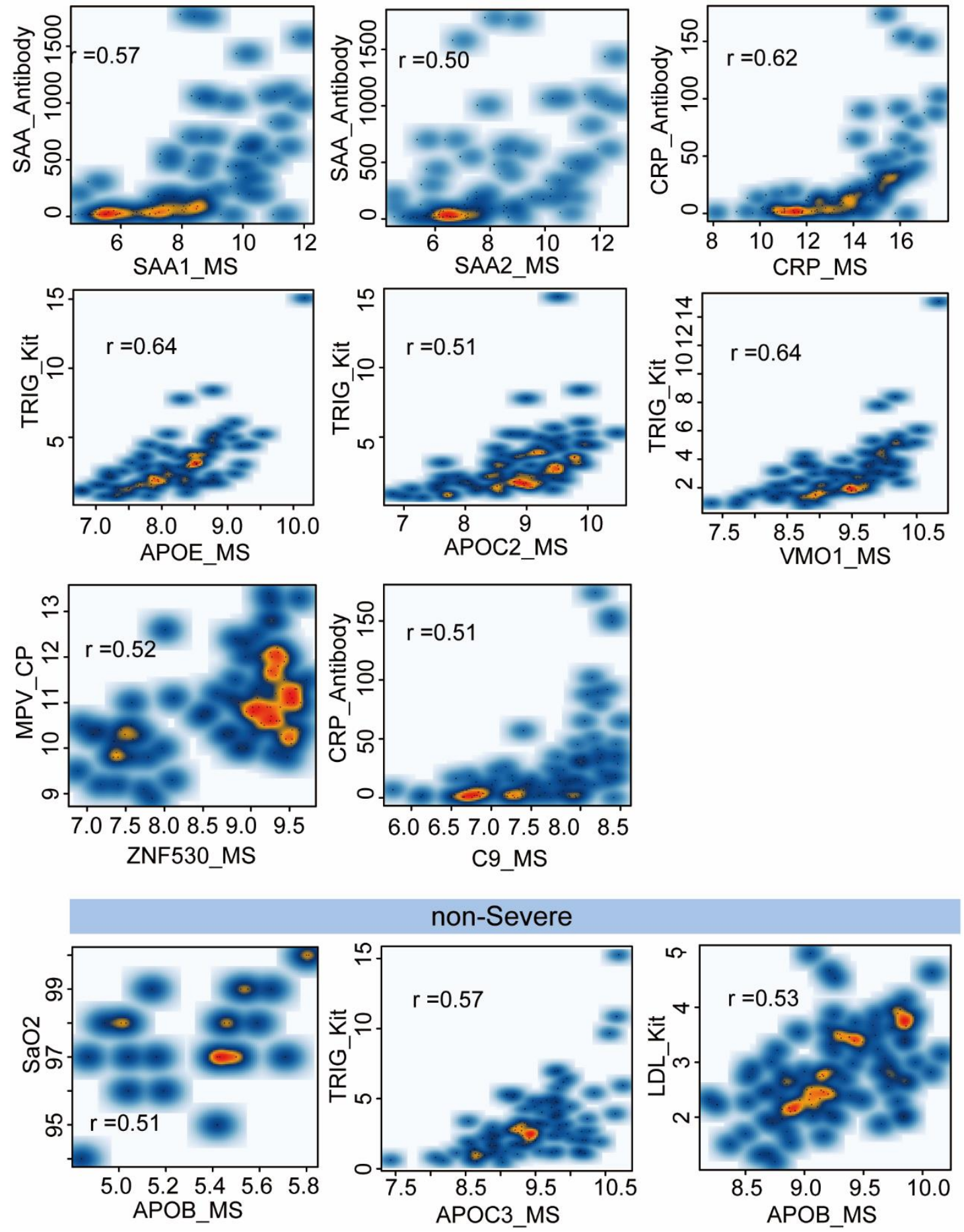
Figure S5. Machine learning using data from 144 patients. Workflow of two machine learning models for predicting the stages of severe and non-severe cases. The models were built using clinical indexes data from 36 severe patients and 108 nonsevere patients.

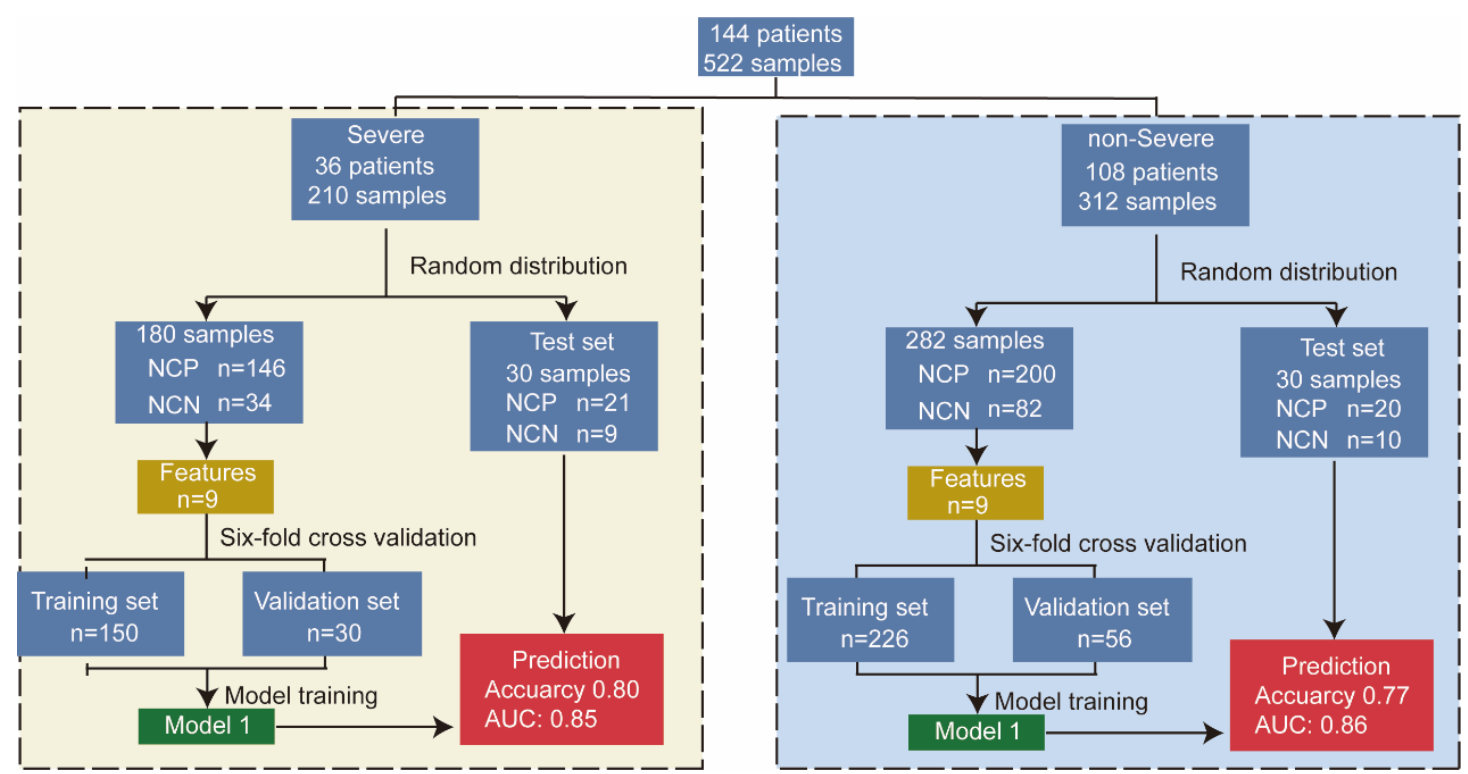


Figure S6. Machine learning using data from 53 patients with proteomic data. Workflow of two machine learning models for predicting the stage of severe and nonsevere cases. The models were built using clinical indexes data from 19 severe patients and 34 non-severe patients.

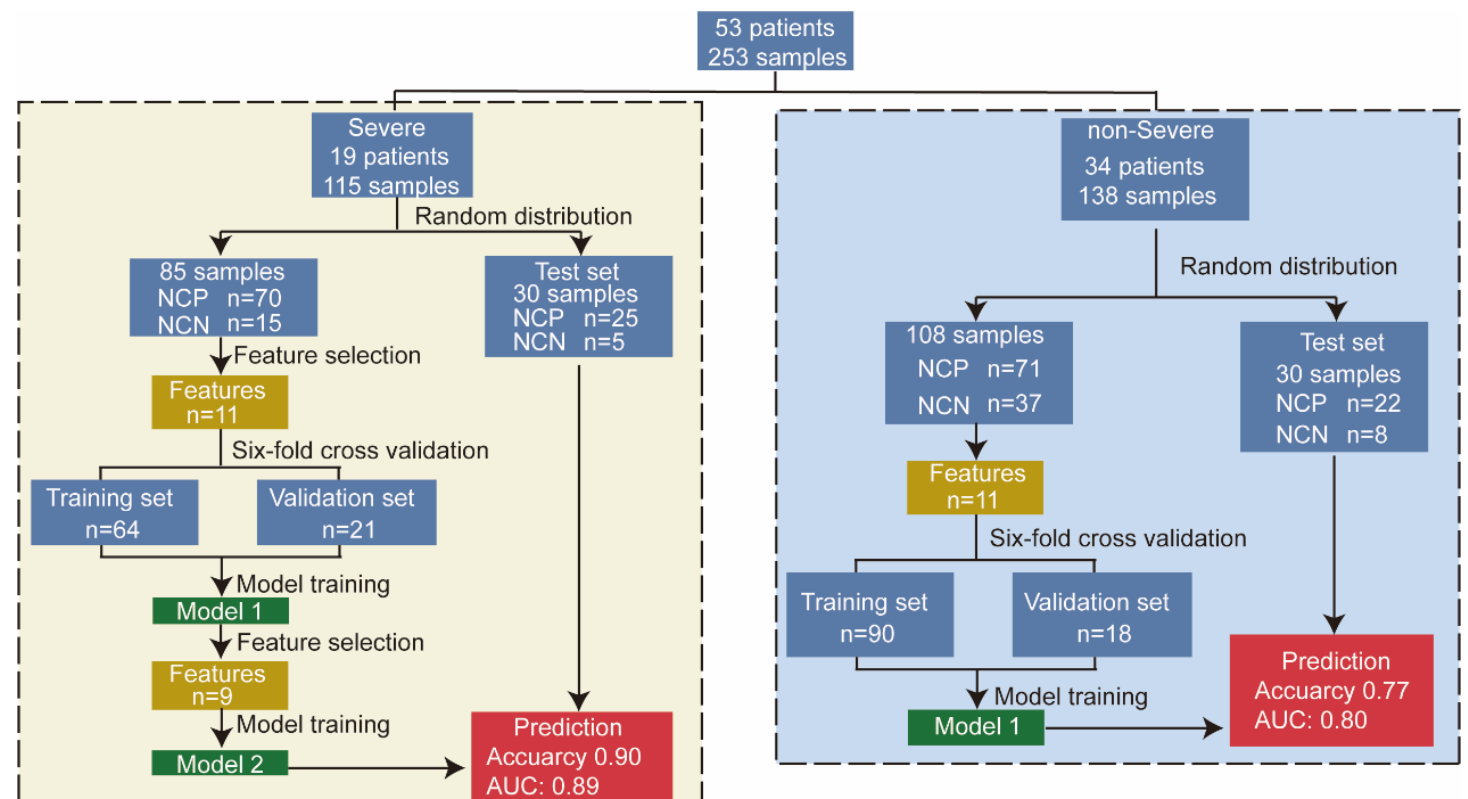

